\title{
An Overview of Professor Yin Kejing's Thinking on the Treatment of Ischemic Apoplexy by TongDu XingNao Acupuncture
}

\author{
Yarong Peng*, Xu Du, Kejing Yin \\ Shaanxi University of Traditional Chinese Medicine, Xianyang 712046, China.
}

How to cite this paper: Yarong Peng, $\mathrm{Xu}$ Du, Kejing Yin. (2021) An Overview of Professor Yin Kejing's Thinking on the Treatment of Ischemic Apoplexy by TongDu XingNao Acupuncture. International Journal of Clinical and Experimental Medicine Research, 5(3), 421-424.

DOI: 10.26855/ijcemr.2021.07.030

Received: June 28, 2021

Accepted: July 20, 2021

Published: August 2, 2021

*Corresponding author: Yarong Peng, Shaanxi University of Traditional Chinese Medicine, Xianyang 712046, China.

Email: 1242883232@qq.com

\begin{abstract}
This paper summarizes Professor Yin Kejing's axial-center theory and experience of the integration of Du meridian, brain marrow, yuan and spirit from the aspects of traditional Chinese medicine classics, basic theory of traditional Chinese medicine, the application of acupuncture in clinical acupoint selection and the timing of stroke wind treatment, etc. The basic theory of this method is sufficient and reliable, and the acupoint selection is less precise and all points are relevant. Starting from the perspective of Yin and Yang and the Five Elements, Qi and blood of the meridians are interlinked to generate blood. Qi and blood are connected to generate vitality of the body, which provides a good and feasible clinical diagnosis and treatment idea for ischemic stroke. Professor Yin put this thinking to good use in the treatment of ischemic stroke. The symptoms of the patients were alleviated successfully and the quality of life of the patients was improved to a large extent. The clinical treatment effect was good.
\end{abstract}

\section{Keywords}

Ischemic Stroke, Acupuncture Treatment, Experience of Famous Doctors, Yin Kejing

Professor Yin Kejing is a national famous acupuncture expert and a national famous old Chinese medicine practitioner. The second, fifth and sixth batch of senior Chinese medicine experts' academic experience inheritance work as a tutor, and the Chinese Academy of Sciences' Chinese Medicine teacher mentor. A career in professional teaching, clinical work and scientific research of traditional Chinese medicine for nearly 60 years, devoting in multifarious pain to heal all sufferings, broad knowledge, in acupuncture treatment of various diseases especially emergency has insight into unique, he in huangdi neijing, herbal medicine preparation and other works of analysis, probe for ischemic stroke, come to the conclusion that a good save and easy diagnosis system. In this paper, Yin Lao's thoughts, insights and clinical experience are summarized as follows.

\section{Research background}

Stroke is an acute cerebral blood circulation disorder, which has the highest morbidity and fatality rate in China. It has the characteristics of high morbidity, high recurrence rate, high disability rate, high mortality rate, low awareness rate, low vacancy rate and low cure rate, which are four high and three low. The main clinical types are hemorrhagic stroke (intracerebral hemorrhage or subarachnoid hemorrhage) and ischemic stroke (cerebral ridge obstruction and cerebral embolism), and the incidence rate of ischemic stroke is up to $87 \%$. According to the latest epidemiological data statistics, the onset age of the disease is getting younger in recent years. 


\section{Construction of TCM theory}

(1) Cerebral stroke is a disease in the brain. The primary pathogenesis is nothing other than cerebral vascular obturation, which leads to cerebral dysfunction. According to Compendium of Materia Medica Xinyi Invention, the brain is the "mansion of the Yuan God". "Yuan" is the first, moving end; "Yuanshen" refers to the activity function of the higher center. Liu Yiming in the "compass" also said: "The god is not thinking of God, can be compared with Yuan Shi shoulder, is the so-called... Yuan god also" [1]. "Fu" means the place where things live and move. "Yuan God's House" means the brain is the place where the higher center of the human body lives and moves. Pivot, the theory of sea again yue: "brain for myeloid sea, it is on the cover and in the house, medullary sea more than the light strength more force, ever since their degrees, not for the brain to turn tinnitus, shin acid being dizzy, overstepping can see, the inactive repose” [2], says brains spilled blood storage energy to compensate the loss of vital qi in order to sustain seeing and hearing, speech, and even movement is normal, to avoid disease; Wang Ang also stated in his book "Notes on Materia Medica" that "human's cognitive ability and memory are both originated in the brain and stored in the brain". It is recorded in the Secret Book of Tricks that "holding the five gods in the brain can regulate the internal organs and regulate the Yin and Yang, and regulate the limbs and bones" [3]. It shows that the brain plays an important role in regulating the activities of qi, blood, Yin, Yang and mental cognition.

(2) Traditional Chinese medicine believes that the heart and brain are connected. According to Su Wen Ling Lan's Secret Essays, "The mind is the official of the monarch, and the god is the official” [4]. It explains that both the mind and the brain can mediate people's spirit, thoughts and thinking. Zhang Xichun, in his Book of Medical Principles, said, "The gods of human beings have physical functions. The body of the gods is stored in the brain, and the use of the gods is in the heart" [5]. A more profound analysis of the heart and brain there is a significant body-use correlation.

(3) The Du meridian follows the middle line of the back of the human body, which is the "sea of Yang meridian". The Du rate the Yang Qi of the human body, and the meridian Qi of the Yang meridian of the three Yang meridian, the belt meridian and the eight strange meridians are connected with it. "Yang Qi is like the sun, if it is lost, life will be shortened and not visible” [6]. The promotion of Yang Qi can promote the growth and development of human body, stabilize the function of zang-fu organs, drive out evil spirits, and promote the early recovery of diseases. The warm and warm function of Yang Qi can maintain the transformation, distribution and metabolism of vital qi and blood fluid, and can help repair damaged organs after disease. Du meridian also by its Du rate a Yang Qi work and brain connection through. On the basis of this theory, Professor Yin put forward the axial theory of the integration of Du meridian, brain marrow and yuan and god to enlighten the mind.

\section{Deep analysis of clinical acupoints}

Acupuncture can regulate the information transmission between glial cells and neurons, improve the blood supply of brain tissue, repair the protective effect of neurons on brain function, improve the number density of synapses after cerebral ischemia to varying degrees, and enhance and promote the excitability of cerebral cortex in stroke patients.

Baihui Point: Baihui Point is the place where the meridians of the three Yang of the hand and the three Yang of the foot converge, and also the place where the Qi and blood of the liver, heart, spleen, lung and kidney sublimate and condense [7]. Foot jue Yin liver meridian go up to the top of the Baihui point and the brain, the liver soul, hide soul, like a bar and evil depression, qi to the brain as the center of the four limbs up to the bones, the soul is also collected in the brain and the main spirit, to play luck and blood flirting with the treatment of chronic diseases. So Baihui can be the main disease, should be four off, Qingfeng Kaiqiao, Tong Du Xingnao, is the first choice of different power points stroke, dredge 100 will meridian qi, a oriaotong is all the oriaways, meridians as the core of acupuncture, a "pass" word to write all the rules of meridians, make the meridians lively.

Shenting Point: It is the vestibule of the mind, the place where human deities, thoughts and cognition exist and move. It is said in Huainan Zi that "God is the deep place of wisdom" [8]. The court of god has the irreplaceable function of concentrating on the mind and regulating the spirit.

Touwei Point: It is the place where the three focal points of Shaoyang in the hand, the gallbladder of Shaoyang in the foot, the stomach of Yangming in the foot, and the meridians and qi of Yangwei converge and gather. The Yang of Yangwei, as the name implies, protects the Yang of the human body, and has the function of maintaining and connecting the Yang meridians of the human body, and overflowing and storing qi and blood. Yangwei and Du intersection in the mute and Feng Fu two points, eventually into the brain, with a live brain Qi blood, promote the recovery of brain vitality. 
Naohu Point: Naohu point means the portal of the brain. The introduction of the brain on the Du meridian is the level of the pulse qi luo brain. It is located in the pillow, where the foot sun meridian meets the pulse qi. Zhang Jingyue mentioned in the "kind of meridian acupuncture class": "the brain, ... It is condensed by the essence of Yuanyang [9]. Therefore, acupuncture on the brain can clear the brain, open the orioles, calm the liver and quench the wind.

Houxi Point: belonging to 8 SEC point of arteries and veins, follow through on hand the meridians to the big job and du meridian are interlinked, the sun for the three Yang, sun be the spirit of flood and grand, du meridian is ZhuYang master plan, as the previous say, people have the sun be the spirit is life without the sun be the spirit is dead, Yang, born of the sun be the spirit by acupuncture si 3 point to mobilize the human body, sun be the spirit of adjustment of grid cloth body, hot fur to spill blood and bones and muscles. Its meaning and the above points have the same idea.

Taichong is the original acupoint of the foot jeyin liver meridian. The original acupoint is the acupoint of the twelve meridians near the wrist and ankle joint, which is the place where the original qi of the viscera is left by the way. According to the Book of Difficulties: Eight Difficulties, "The so-called source of anger is the root of the twelve meridians and the movement of qi between the kidneys" [10]. The original qi starts from the movement of qi between the kidneys, which is the most fundamental driving force of human life activities. By relying on the tri-coke, the whole body runs smoothly, and the upper and lower propagandize, the gasification function of the human body is promoted to realize, and the pulse and pulse are smooth. Yongquan is foot Shaoyin kidney through the well point, is the original gas out of. Liver and kidney two channels, blood and water and wood phase; The original qi in the liver and kidney two meridian has entered, has stopped, has out, causes liver and kidney blood to crisscrosse and stimulate each other, symbiosis sends altogether. Yongquan bearing the sun of the Yang in this, a day of water, underground gush, follow the down and up, soft slow liver Yu, Wang hair Jing Jin to wake up the mind.

\section{The establishment and application of the method of regulating and awakening the brain}

(1) In the field of traditional Chinese medicine, stroke is called stroke, which is caused by the imbalance of Yin and Yang and the disorder of Qi and blood, with sudden fainting, unconsciousness, hemiplegia and mouth and eye askew as the main symptoms [11]. Traditional Chinese medicine divides it into acute period, convalescence period and positroph period. Yin Lao believes that the application of this method to the treatment of stroke is particularly worth paying attention to the problem of treatment timing, early correct diagnosis and effective treatment can greatly reduce the rate of disability of ischemic stroke.

(2) Early interventional treatment: "Suqian Four Qi Regulating the Spirit Theory" "Saints do not cure diseases but not diseases, do not cure chaos but not chaos, this is also called" [12]. For ischemic stroke, if the initial symptoms appear, dizziness, numbness and pain in limbs and body, accompanied by vomiting, insomnia and other symptoms, interventional treatment can be started to treat its precursor; On the basis of the above main point appear dizziness have a headache can add the needle on the sun, star, upper limbs numb limbs can add needle different work point (hand three mile and li 11 attachment point), lower limb numbness and needle leg clearance, blood cupping, insomnia and insomnia thrust needle (about 1 inch needle before the meeting, one side open 2 inch needle), this is not intended for evil to the disease. Yin thought that the early application of Xingnao TongDu Acupuncture could effectively improve the blood supply of ischemic brain tissue, rapidly improve the cerebral oxygen metabolism in the reversible stage of brain tissue injury, reduce the volume of cerebral infarction, exert a positive effect on the inhibition of apoptosis of brain cells, and play a good protective role on ischemic brain tissue. "Seventy-seven Difficulties" said, "The so-called cure for a person without a disease, when he sees a disease of the liver, he should tell it to the spleen. Therefore, he should first check his temper and not cause him to suffer the evil of the liver" [13]. Both disease prevention, before the irreversible brain tissue damage, the use of Xingnao Tongdu needle to regulate Yin and Yang stimulation of cell activation, will tend to deteriorate the tissue into normal. Early acupuncture treatment based on this method can effectively prevent the damage of viable brain tissue in the ischemic penumbra area of brain tissue, minimize the loss of brain tissue function, reduce the incidence of sequelae and promote recovery.

(3) Late adjustment: The convalescence period of ischemic stroke and the afterlife period should not be taken lightly. In the late stage of the disease, qi and Yin deficiency, liver and kidney deficiency, wind phlegm and blood stasis blocking collaterals, no water boat, viscera are not adjusted, and limbs are not used. In order to promote the recovery of the disease, to prevent the disease repeatedly, deterioration, it is necessary to have the five zang with the treatment, dispelling wind and phlegm, nourishing qi and nourishing Yin, regulating the Du meridian, awakening brain and opening the orifices, making up the deficiency and excess, so that the Yang Qi will be in the brain, the blood flow in the brain, smooth function. The liver hides the blood, in the body, the blood nourishes the muscles 
and veins, the kidney hides the essence, in the body, the essence fills the bone marrow. Nourishing the liver and kidney can help the brain to dredge blood vessels, transport sufficient blood, regulate blood volume, prevent bleeding, nourish muscles and bones, prevent vascular sclerosis, eliminate fatigue, avoid the function of fatigue. The heart is the main blood vessel, the heart beats to deliver blood, and the heart is used to stabilize the speed of blood circulation. The spleen is the acquired origin, transporting water and grain, and is the root of transforming Qi and blood. The Qi and blood come from the spleen and stomach, covering the heart and lungs, dispersing the whole body. The central soil is irrigated along the four sides, rising Qing and eliminating phlegm. And the spleen is the main blood, can prevent blood overflow outside the pulse, ensure the flow channel is smooth, so that the brain blood supply is full. The lung regulates the movement and distribution of fine blood along with the rise and fall of qi, and the water and grain transferred by the spleen move the whole body to the fur, facilitate the joints, improve the function, and also help the heart to circulate blood, promote and regulate blood. Keep blood, can moisten the liver, liver blood foot, kidney essence is charge, the essence of the kidney is inflated solid, so that the temper rise, spleen soil Ning, lung gold also benefit. Adopts the method of multi-target, multi-link, multi-effect, multi-way five zang zang harmonization, plays the effect of awakening the brain and regulating the head, so that the qi of the zang fu-qing Yang is transferred to the brain and filled with the brain.

\section{Development prospects and existing problems}

Although Xingnao Tongyu method has been applied in clinical practice and has a good effect on ischemic stroke, it lacks complete, systematic, standardized high-level, high-quality and reliable evidences of evidence-based medicine. The lack of theoretical innovation and systematic and scientific overall treatment plan is the key scientific problem to be solved. Lack of basic scientific evidence that has a key guiding role in clinical diagnosis and treatment. Prospect: Through years of clinical practice and mechanism research, Professor Yin believes that Xingnao TongDu Acupuncture has excellent efficacy in the acute stage, convalesce stage and positure stage of ischemic stroke. Acupoints are few but precise, simple and complex, and all points are in the right place, so that the functions of viscera and meridian can be generated and exchanged, and the mechanism of disease recovery can be observed.

\section{References}

[1] Liu Hong. (2018). Contribution of “Brain as the House of Yuan Gods” to Deep Psychology [J]. China Modern Distance Education of Traditional Chinese Medicine, 2018, 16(17): 67-69.

[2] Bao, Y. Y. (2012). The historical evolution and syndrome differentiation of encephalopathy in TCM [J]. Clin J Clin Med Med., 2012, 24(11): 1029-1034. (in Chinese)

[3] Wang Mingming, Fei Aihua, Fu Yu, Tao Xiang. Cai Shengchao. (2018). Experience in the treatment of post-stroke depression [J]. Jiangxi Journal of Traditional Chinese Medicine, 2018, 49(11): 22-24.

[4] Decorous, An Hong, Shi-jie Xu. (2018). “Officer” of the monarch and the "officer” of Chiang kai-shek data processing [J]. China medical review, 2018(36): 129-131+143.

[5] Liu Shu, Guan Huibo, Xie Ning. (2005). Discussion on the relationship between the five viscera of traditional Chinese medicine and the incidence of senile dementia [J]. Journal of Traditional Chinese Medicine, 2005(08): 1400-1401.

[6] Gong Shichang, Gong Qingdong. (2013). The importance of Yang qi [J]. Jilin Journal of Traditional Chinese Medicine, 2013, 33(09): 867-868.

[7] Xie Zongliang Cao Yi. (2008). "Best” acupuncture clinical experience [J]. Journal of Traditional Chinese Medicine Clinical Journal, 2008(01): 29-31.

[8] Wei, Z. P. (2011). Clinical effect observation of acupuncture on Du meridian acupoints as the main treatment for apoplexy hemiplegia [D]. Guangzhou University of Traditional Chinese Medicine, 2011.

[9] Liu Li'an, Meng Yue, Wang Yulin, Zhou Liqun. (2019). Study on the theory of myeloid sea and the application of naohu point [J]. Chinese Acupuncture and Moxibustion, 2019, 39(09): 1003-1006.

[10] Qian, H. N. (2015). The theory of Yuanqi in Nanjing and its clinical implications [J]. China Journal of Basic Medicine of Traditional Chinese Medicine, 2015, 21(06): 629-630+636.10. (in Chinese)

[11] Xiang Qiao. (2017). Clinical study on the treatment of apoplectic hemiplegia by acupuncture with acupoint method of “Qi lifting and regulating spleen and stomach” [D]. Guangzhou University of Chinese Medicine, 2017.

[12] Liu Jingfeng, Yang Jie, Tang Jun. (2018). Journal of Liaoning University of Traditional Chinese Medicine, 2018, 20(07): 151-154. (in Chinese)

[13] Zhu Xiangdong, Li Guangyuan, Liu Jia, Cheng Changhe. (2008). Chinese Journal of Traditional Chinese Medicine, 2008, 26(12): 2725-2727. (in Chinese) 